\title{
Fully endoscopic microvascular decompression for trigeminal neuralgia: technique review and early outcomes
}

\author{
Leif-Erik Bohman, M.D., John Pierce, M.S., James H. Stephen, M.D., \\ SukhmeEt Sandhu, B.A., and John Y. K. LeE, M.D. \\ Department of Neurosurgery, University of Pennsylvania, Philadelphia, Pennsylvania
}

\begin{abstract}
Object. Fully endoscopic microvascular decompression (E-MVD) of the trigeminal nerve was initially described more than 1 decade ago, but has not yet gained wide acceptance. The authors present the experience of their first 47 consecutive E-MVDs for trigeminal neuralgia (TN).

Methods. All surgeries were performed by a single surgeon (J.Y.K.L.) at the Pennsylvania Hospital at the University of Pennsylvania. Patients prospectively completed pain scales before and after surgery by using the Brief Pain Inventory-Facial outcomes tool. All patients were called on the telephone, and the same outcome tool was administered without reference to their preoperative pain status.

Results. Forty-seven patients (17 men) were identified and enrolled. Forty (85\%) had Burchiel Type 1 TN. Vascular compression was observed at surgery in 42 patients $(89 \%)$. No surgery was aborted or converted to microscope. One patient suffered permanent hearing loss, for a permanent neurological morbidity rate of $2 \%$. Overall improvement in pain outcomes was excellent, with a median maximum pain intensity preoperatively of 10 and postoperatively of $0(\mathrm{p}<0.0001)$. The mean interference with global function scores were 6.2 preoperatively and reduced to 1.0 at last follow-up $(\mathrm{p}<0.0001)$. The mean interference with facial function was 7.3 preoperatively and reduced to 1.2 at last follow-up $(\mathrm{p}<0.0001)$. The mean follow-up period after surgery was $15 \pm 8$ months.

Conclusions. In experienced hands, E-MVD offers superb visualization and illumination and is both safe and effective, at least in the short term. Further longer-term study is needed to compare E-MVD to traditional microscopic MVD.

(http://thejns.org/doi/abs/10.3171/2014.7.FOCUS14318)
\end{abstract}

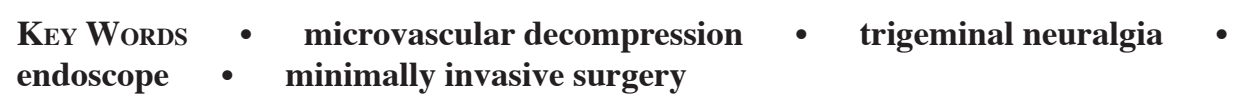

$\mathrm{T}$ HE use of the endoscope has revolutionized anterior or ventral skull base surgery; first as an alternative to the microscope in transsphenoidal pituitary surgery, ${ }^{10}$ and later allowing minimally invasive resections of lesions that are not accessible with a microscopic transsphenoidal approach, with expanded endonasal approaches to the planum sphenoidale, tuberculum sellae, and clivus. ${ }^{19}$ However, use of the endoscope has not yet had a similarly transformative effect on lateral skull base surgery. The advantages of the endoscope over the microscope in cerebellopontine angle (CPA) surgery are the same that it enjoys in endonasal surgery: a panoramic

Abbreviations used in this paper: $\mathrm{AICA}=$ anterior inferior cerebellar artery; BPI = Brief Pain Inventory; CPA = cerebellopontine angle; $\mathrm{E}-\mathrm{MVD}$ = fully endoscopic microvascular decompression; $\mathrm{SCA}=$ superior cerebellar artery; $\mathrm{TN}=$ trigeminal neuralgia . view and unparalleled illumination. The challenges to endoscopic surgery in the CPA are 2-fold: 1) the delicacy of surrounding structures (the cranial nerves, brainstem, and cerebellar hemispheres contrasted with the nasal cartilage, bone, and mucosa); and 2) the lack of a natural access corridor (a potential space lateral to the cerebellar hemisphere contrasted with the aerated sinonasal cavity).

Some of the advantages of endoscopic surgery can be obtained in the CPA by using a hybrid or endoscopeassisted approach..$^{5,22,23}$ Endoscope-assisted techniques require both a conventional microscope and an endoscope. One main disadvantage of endoscope-assisted surgery, however, is that this type of surgery usually relies on the surgeon holding the endoscope in one hand and manipulating neuroanatomy with the other. We believe that endoscope-assisted microsurgery is a valuable adjunct in lateral skull base surgery, but the one-handed surgeon is 
fundamentally restricted in dexterity. Thus, it is only by placing the endoscope on a holding arm that true bimanual, panoramic CPA surgery can be performed. The senior surgeon (J.Y.K.L.) has thus developed a technique for safe, efficient, and fully endoscopic microvascular decompression (E-MVD) of the trigeminal nerve., ${ }^{73}$

We present the results for the first 47 consecutive patients treated with E-MVD of the trigeminal nerve for trigeminal neuralgia (TN). We review our surgical technique as well as short-term results including surgical complications and medium-term outcomes in facial pain measured with a validated facial pain instrument. ${ }^{15}$

\section{Methods}

\section{Data Collection}

All patients undergoing MVD performed by the senior author were enrolled prospectively in an outcomes database. Long-term follow-up was obtained via telephone questionnaire for patients. Patients were assessed using the Brief Pain Inventory (BPI)-Facial, an 18-point validated scale, 3 times throughout their treatment; preoperatively at baseline, a short-term follow-up (within 3 months postoperatively of surgery), and a long-term follow-up (between 6 months and 3 years postoperatively). The BPI-Facial is an adapted version of the BPI, created by the senior author with 7 questions that pertain to specific facial interference. The electronic medical record was retrospectively reviewed to add clinical information to the database. The institutional review board at the University of Pennsylvania approved this study. Short-term results for a subgroup of the patients in this study have been previously reported.?

\section{Patient Selection}

All patients had clinically diagnosed TN and underwent surgery at Pennsylvania Hospital (University of Pennsylvania Health System).

\section{Surgical Technique}

General MVD Technique. The patient's positioning and opening are similar to previously described techniques, and only key aspects of the procedure specific to fully endoscopic surgery will be reviewed. ${ }^{17}$ All operations are performed after induction of general anesthesia. Prior to positioning, the patient's head is secured in a Mayfield clamp. The patient is positioned fully lateral, and neuronavigation is not necessary. In addition, lumbar drains or lumbar puncture are not used. Anatomical landmarks are identified both before skin incision and after muscle dissection and include the following: the tip of the mastoid process and the insertion of the posterior belly of the digastric muscle (approximate course of the sigmoid and transverse sinus). The digastric notch is a useful bony anatomical landmark, whereas the mastoid emissary vein is an inconsistent landmark. A single bur hole is placed, and this is expanded toward the junction of the sigmoid and transverse sinus. For a fully endoscopic procedure, a 1 -cm-diameter " $C$ "- or " $U$ "-shaped dural incision is cre- ated (see Fig. 1). The dura mater is flapped toward the sigmoid sinus with a single stitch.

The first step in the procedure is to dissect the arachnoid and release CSF. Careful, steady, gentle retraction of the cerebellum with a cottonoid patty allows for gradual CSF egress, especially once the medial CPA cistern arachnoid or cerebellomedullary cistern arachnoid is opened. A brain retractor is never used for this surgery because it leads to focal cerebellar ischemia and serves as a physical barrier to restrict movement in an already crowded space. The trigeminal nerve is then identified, inspected, and decompressed. When vascular compression was identified, a Teflon pledget was placed between the vessel and the trigeminal nerve after carefully dissecting the arachnoid around the vessel to fully mobilize it. In cases in which no obvious vascular compression is identified, or in which the compression is primarily venous without significant nerve distortion, an internal neurolysis is performed with a round knife without nerve section. ${ }^{16}$ The dura is closed in a watertight fashion, supplemented with muscle autograft as necessary. The bone is closed either with titanium plate or bone cement, and appropriate layers are closed. Patients stay in the hospital for 2 days on average, and postoperative imaging is not necessary.

Specifics of $E-M V D$. An endoscope holder is used to allow bimanual dexterity, to stabilize the endoscope to prevent unintended injury of the surrounding neurovascular structures, and to allow adjustment of trajectory throughout the case. A Mitaka Pneumatic Arm (Mitaka Kohki Co.), which is firmly secured to the bed, was used for all cases. ${ }^{9}$ We generally use a $2.7-\mathrm{mm}$ outer diameter offset endoscope (Storz). The smaller diameter of this endoscope as opposed to the standard 4-mm sinus endoscope maximizes the available working space for the other instruments, minimizing both instrument clash and brain retraction. A high-definition camera (1080 pixels) and screen are used to maximize image quality. Light intensity is kept to $25 \%-50 \%$ on the Storz system to minimize the possibility of thermal injury to adjacent neural structures, and no irrigating sheath is used.

Further details of our surgical technique have been previously published. ${ }^{13}$ Instrument and endoscope clash in the limited confines of the CPA represent the major technical challenge to E-MVD, in our experience. By maintaining a strict triangle in the introduction and placement of endoscope and right- and left-hand instruments, the confusion and conflict of instruments within the tight confines of the CPA is minimized. The endoscope is parked at the apex of the visualization triangle, usually along the petrous dura/temporal bone/sigmoid sinus. The endoscope must be located at the apex of the triangle both proximally (dura) as well as distally (petrous temporal bone). There is both a distal and a proximal triangle. At the entrance of the cerebellar convexity dura, the endoscope must be at the apex at "12 o'clock," so that instruments can be passed underneath the endoscope along the cerebellar folia surface at "5 o'clock" and "7 o'clock." Next, there is a distal triangle, which is visualized on screen. Of note, when working closely on the trigeminal nerve, some portion of the eighth cranial nerve is always kept in view 


\section{Fully endoscopic microvascular decompression for TN}

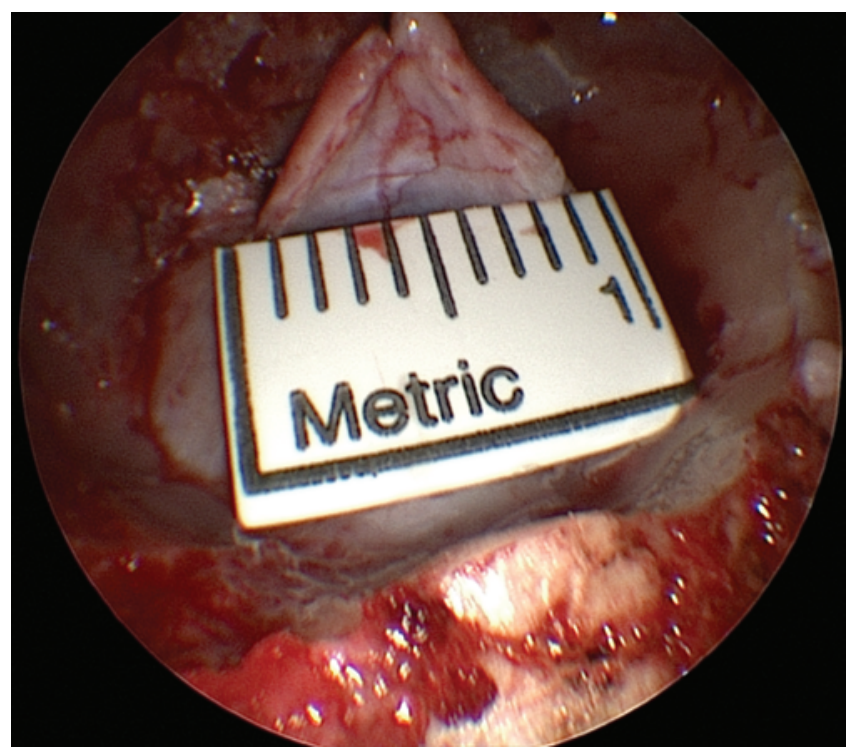

Fig. 1. Endoscopic view of craniectomy for E-MVD of the trigeminal nerve. The dura mater has been opened and reflected toward the sigmoid sinus. A cut section of ruler has been placed over the cerebellar hemisphere for reference.

when "parking" the endoscope. This allows instruments to be introduced under the endoscope without the need of other adjuncts such as a micromanipulator. By visualizing where the instruments should appear within the field before they arrive on screen and by confirming that the instruments emerge at the appropriate position within the distal "triangle," conflicts are avoided. For example, if the endoscope is understood to be on the petrous face at the "12 o'clock" position, the left-handed 5-Fr suction should emerge on screen at "7 o'clock," and the bipolar/dissector should emerge on screen at "5 o'clock."

The majority of the initial surgery is performed with the $0^{\circ}$ endoscope. The "triangle method" to fully endoscopic CPA surgery does allow excellent dissection at the base of the triangle, including the root entry zone of the cranial nerves. Indeed, there is no difficulty with dynamic retraction of the cerebellum using the left-handed suction and microdissection of the root entry zone with the right hand using standard microsurgical techniques. Indeed, a $30^{\circ}$ medially (down) directed endoscope provides excellent visualization of the root entry zone of the facial nerve for hemifacial spasm. This technique eliminates any need for a retractor along the flocculus of the cerebellum. The triangle method, however, does make it difficult to perform the dissection distally along the trigeminal nerve out toward the Meckel cave. Although the classic teaching is that compression is located at the root entry zone along the Obersteiner Redlich zone, the senior author has identified venous compression distally as well as arachnoid distortion and displacement of the nerve in the distal location near the entrance into the Meckel cave. Curved instruments can be used to assist with dissection in this area.

\section{Statistical Analysis}

Statistical analyses were performed using Stata ver- sion 11.2 (Stata Corp.). Chi-square tests were used for categorical variables. For analysis of general and facial BPI values, for individual items, differences between the 2 groups were tested using the Wilcoxon rank-sum test for nonparametric measures. Within an individual factor or domain, the individual scores were summed and averaged for a mean score. Differences between the 2 groups were tested using a Student t-test for parametric measures.

Of note, because this is a short-term outcome study with only 15 months of follow-up time, we chose not to perform Kaplan-Meier statistics. Survival curves are best for studying the amount of time to an event (usually death). These curves have been applied to the field of pain in TN with the assumption that patients experience a certain amount of time to their event (recurrence of TN). Because our study did not have enough follow-up time to demonstrate a time to event, we chose to measure pain before surgery on a $0-10$ scale by using the BPI-Facial, and then to measure pain after surgery at the last follow-up time by using the same BPI-Facial outcomes tool.

\section{Results}

Between September 2010 and July 2013, 105 patients underwent MVD performed by the senior author for cranial nerve disorders including $\mathrm{TN}$, hemifacial spasm, geniculate neuralgia, and glossopharyngeal neuralgia at Pennsylvania Hospital. Only the 90 patients who underwent MVD for TN were included in this analysis. During this study period, 23 patients underwent MVD with a microscope only, and 9 underwent endoscope-assisted MVD. The remaining 58 patients underwent purely endoscopic MVD. This transition from MVD to E-MVD has been detailed in a prior paper from our group. ${ }^{7}$ In the last full year, from August 2012 to July 2013 (representing 35 cases), microscopic MVD was only performed in 1 case because the endoscope-holding arm was not physically available. This absence was identified prior to the start of surgery. This study focuses purely on the patients who underwent endoscopic MVD and in whom follow-up data were available -47 of the 58 patients who underwent MVD.

The median age at surgery was 60 years (range 27-77 years). Thirty patients (64\%) had right-sided symptoms, and no patients had bilateral symptoms in this series. Six patients $(13 \%)$ had undergone prior interventional treatment for TN on the ipsilateral side. The majority of patients had V2 or V3 distribution of pain, or both, and 40 (85\%) had Burchiel Type 1 TN. The median duration of symptoms prior to surgery was 5 years (range 1 month-27 years). Details of patient characteristics including prior treatments are provided in Table 1.

Vascular compression was noted at surgery in 42 patients (89\%). The superior cerebellar artery (SCA) was the most commonly identified single vessel, but other arteries, veins, and artery/vein combinations of compression were observed (Table 2). The endoscope offered superb visualization of vascular compression (Figs. 2-4). The median length of stay was 3 days (range $2-15$ days). There were no perioperative deaths. Dizziness/unsteadiness without focal neurological signs was the most common complica- 
TABLE 1: Characteristics in 47 patients with TN

\begin{tabular}{|c|c|}
\hline Characteristic & Value (\%) \\
\hline total no. of patients & 47 \\
\hline \multicolumn{2}{|l|}{ age in yrs at op } \\
\hline median & 60 \\
\hline range & $27-77$ \\
\hline no. of males & $17(36.2)$ \\
\hline \multicolumn{2}{|l|}{ site of op } \\
\hline It side of face & $17(36.2)$ \\
\hline rt side of face & $30(63.8)$ \\
\hline bilat & $0(0)$ \\
\hline \multicolumn{2}{|l|}{ age in yrs at onset of symptoms } \\
\hline median & 60 \\
\hline range & $24-77$ \\
\hline \multicolumn{2}{|l|}{ prior drug treatment } \\
\hline carbamazepine & $21(44.7)$ \\
\hline phenytoin & $1(2.1)$ \\
\hline oxcarbazepine & $6(12.8)$ \\
\hline neurontin & $11(23.4)$ \\
\hline baclofen & $10(21.3)$ \\
\hline \multicolumn{2}{|l|}{ prior procedures } \\
\hline any procedure & $6(12.8)$ \\
\hline MVD & $4(8.5)$ \\
\hline Gamma Knife radiosurgery & $2(4.3)$ \\
\hline Linac-based radiosurgery & $0(0)$ \\
\hline other & $0(0)$ \\
\hline \multicolumn{2}{|l|}{ distribution of pain } \\
\hline V1 only & $1(2.1)$ \\
\hline V2 only & $11(23.4)$ \\
\hline V3 only & $6(12.8)$ \\
\hline V1 \& V2 & $9(19.1)$ \\
\hline V2 \& V3 & $16(34.0)$ \\
\hline $\mathrm{V} 1, \mathrm{~V} 2, \& \mathrm{~V} 3$ & $4(8.5)$ \\
\hline Burchiel Type 1 TN & 40 (85.1) \\
\hline Burchiel Type 2 TN & $7(14.9)$ \\
\hline
\end{tabular}

tion, occurring in 3 patients (6\%), and other postoperative complications included severe headache and transient blurred vision, which was self-limited. One patient had significant hearing loss on the ipsilateral side to the MVD, and 1 patient had postoperative CSF leakage requiring lumbar drain placement but no additional surgery. The total permanent neurological morbidity rate was thus $2 \%$. Complications are listed in Table 3.

No fully endoscopic procedure was aborted because of instrumentation complications. In addition, no fully endoscopic procedure was converted to a microscopic procedure because of lack of visualization of instrumentation complications.

Preoperatively, 36 patients (77\%) were using medications for neuropathic pain, and only $17(36 \%)$ were using these medications at last follow-up $(\mathrm{p}<0.0001)$. Of note,
TABLE 2: Nerve compression observed at surgery in 47 patients with $\mathrm{TN}^{*}$

\begin{tabular}{lc}
\hline \multicolumn{1}{c}{ Vessel } & No. $(\%)$ \\
\hline SCA & $25(53.2)$ \\
AICA & $5(10.6)$ \\
dolichoectatic VBA & $1(2.1)$ \\
unspecified small artery & $6(12.8)$ \\
vein & $10(21.3)$ \\
vein only & $8(17.0)$ \\
unspecified small artery or vein only & $11(23.4)$ \\
no vessel identified & $5(10.6)$ \\
\hline
\end{tabular}

* $\mathrm{VBA}=$ vertebrobasilar artery.

some of the patients remained on medications such as gabapentin for unrelated pain like radiculopathy. No attempt was made to separate this during the phone questionnaire. The mean length of follow-up for completion of the BPIFacial was 15 months (SD 8 months). Details of the BPIGeneral and BPI-Facial outcomes are given in Table 4. Overall, 39 patients (83\%) rated their pain as "Very Much Improved" or "Much Improved," and 44 patients (94\%) had at least some improvement in their preoperative facial pain. The median patient-rated maximum pain intensity preoperatively (out of 10) was 10, which dropped to 0 at last follow-up ( $\mathrm{p}$ 0.0001). The mean self-rated reduction in pain intensity was $84 \%$. The mean interference with global function scores was 6.2 preoperatively and reduced to 1.0 at last follow-up $(\mathrm{p}<0.0001)$. The mean interference with facial function was 7.3 preoperatively and reduced to 1.2 at last follow-up $(\mathrm{p}<0.0001)$.

\section{Discussion}

Microvascular decompression of the trigeminal nerve is the most durable and efficacious treatment for $\mathrm{TN}^{3}{ }^{3} \mathrm{Un}$ der microscopic visualization, excellent clinical outcomes can be obtained, but using the operating microscope to visualize deep structures has limitations of light and visualization that frequently require a larger bony opening and may require increased retraction of the cerebellum and, thus, traction on the seventh, eighth, and lower cranial nerves. The endoscope offers superb panoramic visualization and illumination. Multiple groups have demonstrate that endoscope-assisted approaches (where the bulk of the procedure is done with the operating microscope but an endoscope is used to inspect the nerve before or after decompression) lead to increasing identification of vascular contact and compression, which were not appreciated with microscopy alone. $1,4,5,5,12,18,20,23$ However, using the endoscope in the CPA sacrifices binocular vision and is technically challenging, with its own learning curve.

First described in 2001 by Eby et al., ${ }^{6}$ E-MVD has now been reported in several single-center series. ${ }^{2,7,11,21,24}$ All report excellent safety and short- to medium-term functional results. The largest of these, by Kabil et al., reported results for 255 patients with a favorable 3-year 


\section{Fully endoscopic microvascular decompression for TN}
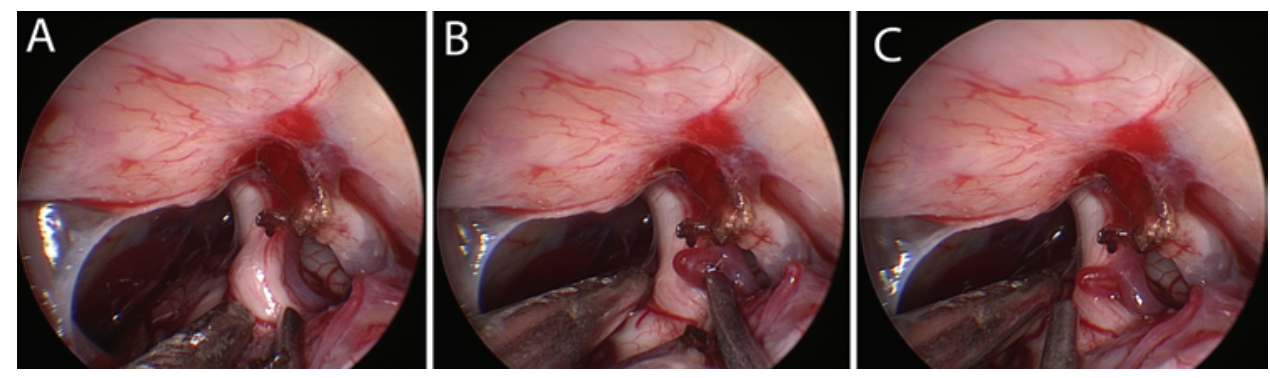

FIG. 2. Left-sided approach demonstrating SCA compression of trigeminal nerve and illustrating the SCA loop that was compressing the rostral side of the nerve, which was dissected out and lifted off the nerve. A Teflon pledget was then placed between the SCA loops and the nerve (not shown).

success rate and a lower rate of complications when compared with published series of microscopic MVD. ${ }^{11}$

In this study we found no obvious vessel compression in as many as $10 \%$ of patients. In a classic paper (Barker et al.), Dr. Jannetta published his life's work. This paper described a series in which MRI was not used to base the decision to go to the operating room, and in which the operating microscope was used exclusively; $75 \%$ of the time the SCA compressed the trigeminal nerve and $10 \%$ of the time the anterior inferior cerebellar artery (AICA) compressed the nerve. However, a vessel was identified in all cases, although $19 \%$ were compressed by a small unnamed vessel. ${ }^{3}$ In another modern-day microscopic surgery series (Lee et al.), Dr. Burchiel published his results. This paper reported that in patients with classic TN who underwent their first surgery with Dr. Burchiel between 2006 and 2013, 26 of 145 patients (18\%) did not have evidence of vascular compression..$^{14}$ Thus, our findings in this study do not differ substantially from the range seen in prior studies.

One of the putative advantages of the endoscope as compared with the microscope is that hidden compression may be more easily identified. Indeed, Teo et al. report that in their series of endoscope-assisted MVDs they identified vascular compression in all patients with the aid of the endoscope. They report that in $8 \%$ of the cases no vessel was identified with the microscope but was identified with the endoscope. ${ }^{23}$ In contrast to this study, a comparison between microscope and endoscope findings could not be performed in our study because the microscope was not draped or prepared for the procedure. One method in which this paper could have been strengthened is if a comparison between the $0^{\circ}$ and $30^{\circ}$ angled scope had been performed. Indeed, in this series the angled endoscope was frequently but not consistently used.

The selection of patients for conventional microscopic MVD versus E-MVD in this series represents a gradual transition from conventional to fully endoscopic by the senior author. Early in the series, the fully endoscopic procedure was easier to perform in 2 groups of patients: the elderly because their brain is more slack, and patients with a small petrous tubercle (suprameatal tubercle). By the end of this series virtually all patients underwent E-MVD. At present, the senior author performs E-MVD exclusively.

The use of the BPI-Facial is a relatively new method to measure pain outcomes in patients with TN. We have found it relatively easy to use with our patients, and it captures both pain intensity and interference with aspects of daily living. The questionnaire consists of 17 questions with a scale from 0 to 10 for each of the questions and is thus easy to administer. By applying the BPI-Facial measurement tool before intervention, this study raises the quality of study design in retrospective studies of pain outcomes. Prior studies of TN have only rarely attempted to measure pain before surgical intervention. In this study we measured pain before and after surgical intervention. In addition, in this type of short-term interim study with relatively short follow-up (15 months), we believe the degree of pain relief that our patients experience is captured in a quantitative manner. Obviously, longer-term studies will be necessary to see whether pain relief remains durable over time.

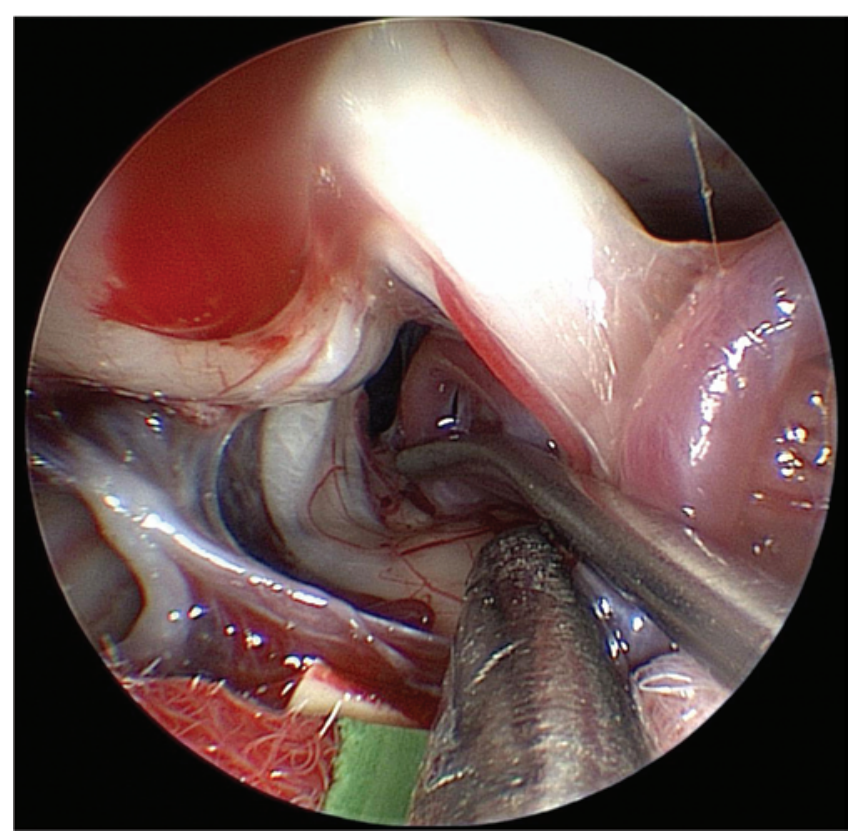

FIG. 3. Left-sided approach demonstrating the seventh/eighth cranial nerve complex in the foreground with a cranial loop of the AICA, which was compressing the trigeminal nerve (AICA loop retracted by the dissector). 

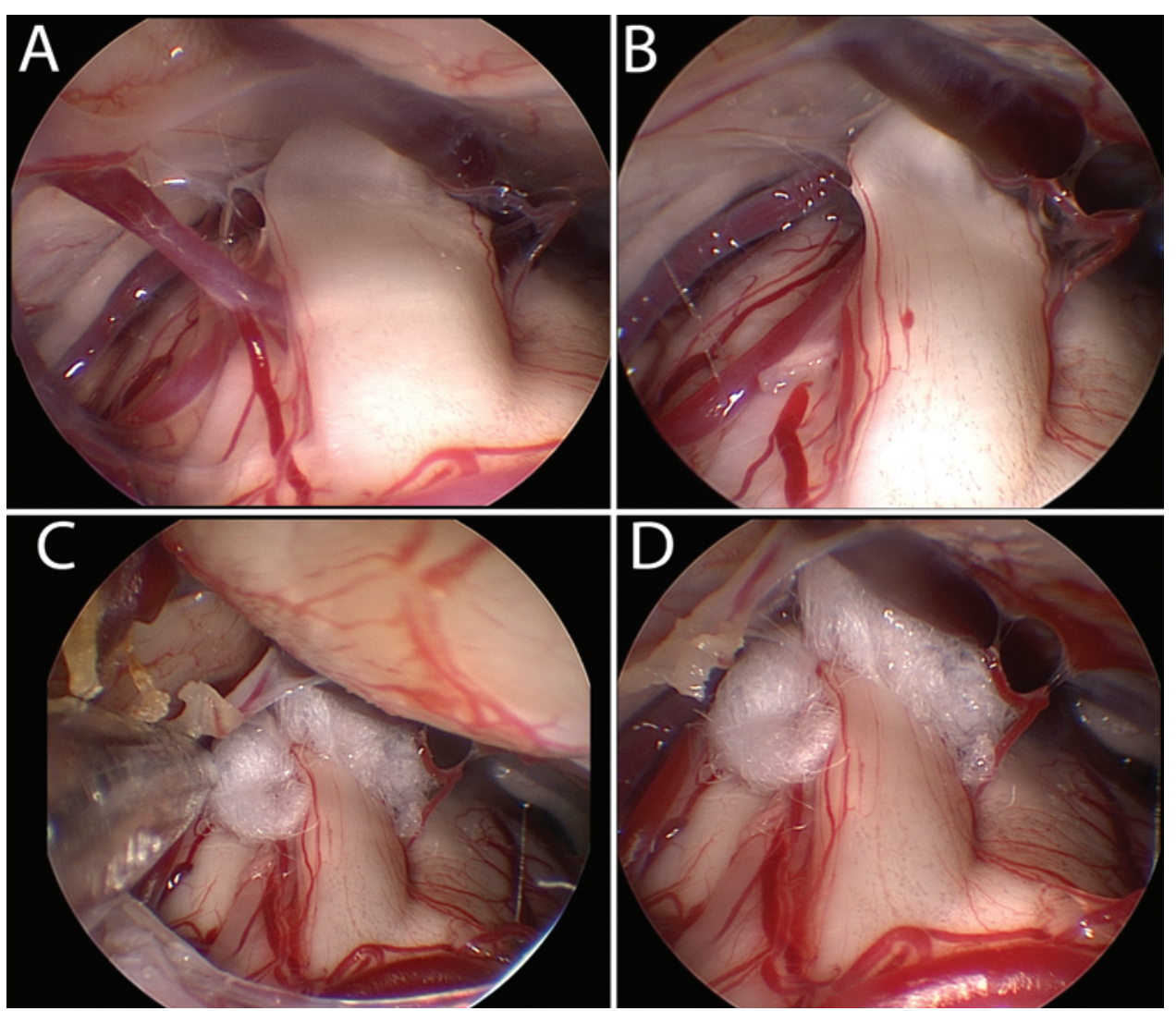

FIG. 4. Endoscopic views demonstrating a right-sided approach with compression of the trigeminal nerve by both a small SCA branch and by a vein. Both vein and artery were mobilized and Teflon pledgets were placed between the vein and artery and the trigeminal nerve.

Trigeminal MVD with the operating microscope has a long history, with excellent long-term results. The EMVD procedure remains in its relative infancy, and we believe that these results suggest an excellent safety profile and medium-term functional results. Our study has

TABLE 3: Incidence of surgical and postsurgical complications in 47 patients who underwent E-MVD

\begin{tabular}{ll}
\hline \multicolumn{1}{c}{ Complication } & No. $(\%)$ \\
\hline death & $0(0)$ \\
corneal numbness & $0(0)$ \\
stroke & $0(0)$ \\
facial paralysis & $0(0)$ \\
paralysis of arms \&/or legs & $0(0)$ \\
hearing loss (permanent) & $1(2.1)$ \\
transient unsteadiness/dizziness & $3(6.4)$ \\
vertigo & $1(2.1)$ \\
headaches & $1(2.1)$ \\
vision problems (transient) & $1(2.1)$ \\
diplopia & $0(0)$ \\
hydrocephalus requiring shunt & $0(0)$ \\
CSF leak & $1(2.1)$ \\
\hline
\end{tabular}

multiple limitations, including retrospective collection of clinical data points and inconsistent length of followup. Moreover, all surgeries were performed by a highly experienced skull base surgeon who is comfortable with both the microscopic approach to MVD and endoscopic approaches in the ventral skull base, which may limit generalizability.

\section{Conclusions}

In experienced hands, E-MVD of the trigeminal nerve is safe and offers excellent clinical outcomes. Further study is needed to see if the advantages of visualization offered by the endoscope translate into superior clinical outcomes compared with traditional microscopic approaches for MVD.

\section{Disclosure}

Dr. Lee received clinical or research support from Storz for the study described and owns stock in VisionSense.

Author contributions to the study and manuscript preparation include the following. Conception and design: Lee. Acquisition of data: Pierce, Sandhu. Analysis and interpretation of data: Bohman, Pierce, Sandhu. Drafting the article: Bohman, Stephen. Critically revising the article: Lee, Bohman, Stephen. Reviewed submitted version of manuscript: Bohman, Pierce, Stephen. Approved the final version of the manuscript on behalf of all authors: Lee. Study supervision: Lee. 


\section{Fully endoscopic microvascular decompression for TN}

TABLE 4: Functional outcomes based on BPI scores in 47 patients with TN

\begin{tabular}{lccc}
\hline \multicolumn{1}{c}{ Parameter } & Baseline & Last Follow-Up & $p$ Value \\
\hline median severity of pain score $(n=41)$ & & \\
pain at its worst & 10 & 0 & $<0.0001^{*}$ \\
pain at its least & 1 & 0 & $0.0006^{*}$ \\
median interference $w$ / general function score $(n=43)$ & \\
activity & 8 & 0 & $<0.0001^{*}$ \\
mood & 8 & 0 & $<0.0001^{*}$ \\
walking & 3 & 0 & $<0.0001^{*}$ \\
work & 8 & 0 & $<0.0001^{*}$ \\
relationships & 7 & 0 & $<0.0001^{*}$ \\
sleep & 7 & 0 & $<0.0001^{*}$ \\
enjoyment of life & 9 & 0 & $<0.0001^{*}$ \\
mean score & 6.2 & 1.0 & $<0.0001 \dagger$ \\
median interference w/ facial function score $(n=43)$ & \\
eating & 9 & 0 & $<0.0001^{*}$ \\
touching & 8 & 0 & $<0.0001^{*}$ \\
brushing & 9 & 0 & $<0.0001^{*}$ \\
smiling & 8 & 0 & $<0.0001^{*}$ \\
talking & 8.5 & 0 & $<0.0001^{*}$ \\
opening mouth & 8 & 0 & $<0.0001^{*}$ \\
hard foods & 10 & 0 & $<0.0001^{*}$ \\
mean score & 7.3 & 1.2 & $<0.0001^{\dagger}$ \\
\hline
\end{tabular}

* Wilcoxon signed-rank test for paired nonparametric values.

$\dagger$ Student t-test for comparison of paired parametric values.

\section{References}

1. Abdeen K, Kato Y, Kiya N, Yoshida K, Kanno T: Neuroendoscopy in microvascular decompression for trigeminal neuralgia and hemifacial spasm: technical note. Neurol Res 22: $522-526,2000$

2. Artz GJ, Hux FJ, Larouere MJ, Bojrab DI, Babu S, Pieper DR: Endoscopic vascular decompression. Otol Neurotol 29:9951000,2008

3. Barker FG II, Jannetta PJ, Bissonette DJ, Larkins MV, Jho HD: The long-term outcome of microvascular decompression for trigeminal neuralgia. N Engl J Med 334:1077-1083, 1996

4. Charalampaki P, Kafadar AM, Grunert P, Ayyad A, Perneczky A: Vascular decompression of trigeminal and facial nerves in the posterior fossa under endoscope-assisted keyhole conditions. Skull Base 18:117-128, 2008

5. Chen MJ, Zhang WJ, Yang C, Wu YQ, Zhang ZY, Wang Y: Endoscopic neurovascular perspective in microvascular decompression of trigeminal neuralgia. J Craniomaxillofac Surg 36:456-461, 2008

6. Eby JB, Cha ST, Shahinian HK: Fully endoscopic vascular decompression of the facial nerve for hemifacial spasm. Skull Base 11:189-197, 2001

7. Halpern CH, Lang SS, Lee JY: Fully endoscopic microvascular decompression: our early experience. Minim Invasive Surg 2013:739432, 2013

8. Jarrahy R, Berci G, Shahinian HK: Endoscope-assisted microvascular decompression of the trigeminal nerve. Otolaryngol Head Neck Surg 123:218-223, 2000
9. Jarrahy R, Eby JB, Shahinian HK: A new powered endoscope holding arm for endoscopic surgery of the cranial base. Minim Invasive Neurosurg 45:189-192, 2002

10. Jho HD, Carrau RL: Endoscopy assisted transsphenoidal surgery for pituitary adenoma. Technical note. Acta Neurochir (Wien) 138:1416-1425, 1996

11. Kabil MS, Eby JB, Shahinian HK: Endoscopic vascular decompression versus microvascular decompression of the trigeminal nerve. Minim Invasive Neurosurg 48:207-212, 2005

12. King WA, Wackym PA, Sen C, Meyer GA, Shiau J, Deutsch H: Adjunctive use of endoscopy during posterior fossa surgery to treat cranial neuropathies. Neurosurgery 49:108-116, 2001

13. Lang SS, Chen HI, Lee JY: Endoscopic microvascular decompression: a stepwise operative technique. ORL J Otorhinolaryngol Relat Spec 74:293-298, 2012

14. Lee A, McCartney S, Burbidge C, Raslan AM, Burchiel KJ: Trigeminal neuralgia occurs and recurs in the absence of neurovascular compression. Clinical article. J Neurosurg 120: 1048-1054, 2014

15. Lee JY, Chen HI, Urban C, Hojat A, Church E, Xie SX, et al: Development of and psychometric testing for the Brief Pain Inventory-Facial in patients with facial pain syndromes. Clinical article. J Neurosurg 113:516-523, 2010

16. Ma Z, Li M: "Nerve combing" for trigeminal neuralgia without vascular compression: report of 10 cases. Clin J Pain 25: 44-47, 2009

17. McLaughlin MR, Jannetta PJ, Clyde BL, Subach BR, Comey $\mathrm{CH}$, Resnick DK: Microvascular decompression of cranial nerves: lessons learned after 4400 operations. J Neurosurg 90:1-8, 1999

18. Miyazaki H, Deveze A, Magnan J: Neuro-otologic surgery through minimally invasive retrosigmoid approach: endoscope assisted microvascular decompression, vestibular neurotomy, and tumor removal. Laryngoscope 115:1612-1617, 2005

19. Paluzzi A, Gardner P, Fernandez-Miranda JC, Snyderman C: The expanding role of endoscopic skull base surgery. Br $\mathbf{J}$ Neurosurg 26:649-661, 2012

20. Rak R, Sekhar LN, Stimac D, Hechl P: Endoscope-assisted microsurgery for microvascular compression syndromes. Neurosurgery 54:876-883, 2004

21. Setty P, Volkov AA, D’Andrea KP, Pieper DR: Endoscopic vascular decompression for the treatment of trigeminal neuralgia: clinical outcomes and technical note. World Neurosurg 81:603-608, 2014

22. Takemura Y, Inoue T, Morishita T, Rhoton AL Jr: Comparison of microscopic and endoscopic approaches to the cerebellopontine angle. World Neurosurg [epub ahead of print], 2013

23. Teo C, Nakaji P, Mobbs RJ: Endoscope-assisted microvascular decompression for trigeminal neuralgia. Technical case report. Neurosurgery 59 (4 Suppl 2):ONSE489-ONSE490, 2006

24. Yadav YR, Parihar V, Agarwal M, Sherekar S, Bhatele P: Endoscopic vascular decompression of the trigeminal nerve. Minim Invasive Neurosurg 54:110-114, 2011

Manuscript submitted June 14, 2014.

Accepted July 24, 2014.

Please include this information when citing this paper: DOI: 10.3171/2014.7.FOCUS14318.

Address correspondence to: John Y. K. Lee, M.D., Department of Neurosurgery, University of Pennsylvania, 235 S. 8th St., Philadelphia, PA 19106.email: leejohn@uphs.upenn.edu. 